\title{
A FAULT TOLERANT DIRECT CONTROL ALLOCATION SCHEME WITH INTEGRAL SLIDING MODES
}

\author{
Mirza TARIQ HAMAYUN ${ }^{a}, *$ Christopher EDWARDS ${ }^{b}$, HALIM ALWI $^{b}$, \\ ABDULRAHMAN BAJODAH ${ }^{c}$ \\ ${ }^{a}$ Department of Electrical Engineering \\ COMSATS Institute of Information Technology, Lahore, Pakistan \\ e-mail: mhamayuneciitlahore.edu.pk \\ ${ }^{b}$ Department of Engineering \\ College of Engineering, Mathematics and Physical Sciences, University of Exeter, UK \\ e-mail: \{c.edwards, h.alwi\}@exeter.ac.uk \\ ${ }^{c}$ Department of Aeronautical Engineering \\ King Abdul Aziz University, Jeddah, Saudi Arabia \\ email: abajodah@kau.edu.sa
}

\begin{abstract}
In this paper, integral sliding mode control ideas are combined with direct control allocation in order to create a fault tolerant control scheme. Traditional integral sliding mode control can directly handle actuator faults; however, it cannot do so with actuator failures. Therefore, a mechanism needs to be adopted to distribute the control effort amongst the remaining functioning actuators in cases of faults or failures, so that an acceptable level of closed-loop performance can be retained. This paper considers the possibility of introducing fault tolerance even if fault or failure information is not provided to the control strategy. To demonstrate the efficacy of the proposed scheme, a high fidelity nonlinear model of a large civil aircraft is considered in the simulations in the presence of wind, gusts and sensor noise.
\end{abstract}

Keywords: fault tolerant control, integral sliding mode control, linear matrix inequalities.

\section{Introduction}

Reliability and safety is an ever increasing requirement in modern engineering, especially in safety critical applications (e.g., fly-by-wire aircraft, drive-by-wire automobiles, etc.). Reliability can be enhanced using fault tolerant control schemes (Wu et al., 2000). A survey of different design methodologies and their comparison in the framework of fault tolerant control (FTC) has been presented by Zhang and Jiang (2008), who argued that while designing an FTC system a trade-off between the achievable performance and available actuator capabilities should be carefully examined to deal with worst case situations. Achieving a performance close to the nominal one and maintaining an overall closed-loop stability in the face of actuator faults/failures as well as in the presence of aerodynamic disturbances (i.e., wind gusts) and sensor noise is the main objective to be met in any FTC design.

* Corresponding author
Recently an active FTC approach has been proposed by Castaldi et al. (2014) for nonlinear aerospace models, which is an extended version of their previous attempt (Castaldi et al., 2011), where a fault detection and isolation (FDI) module is designed via a nonlinear geometric approach, giving fault estimates, analytically decoupled from other faults and disturbances (vertical wind gusts). To maintain closed-loop stability in the case of failures in certain actuators, the control effort can be redistributed to healthier actuators to obtain the desired performance, or at least some level of acceptable performance. To design such a control system, the plant itself must be equipped with redundant control effectors, which can be exploited to achieve fault tolerance (Zhang and Jiang, 2003). The work described by Ducard (2009) and Edwards et al. (2010), for example, provides an overview of the most recent results in the area of FTC for aerospace applications. 
Control allocation (CA) is a technique which has the capability to use redundant control effectors in an efficient way in order to achieve fault tolerance without the need to reconfigure the underlying control law (Davidson et al., 2001). The advantageous feature of CA is that the underlying "virtual" control effort can be created using any suitable control paradigm. In this context, CA can be viewed as a mechanism for distributing a lower order virtual control signal generated by an appropriate control paradigm to the physical actuator (Harkegard and Glad, 2005; Bošković and Mehra, 2002) to affect FTC. A recent survey of different $\mathrm{CA}$ algorithms for a range of engineering applications is documented by Johansen and Fossen (2013).

Sliding mode control (SMC) (Utkin et al., 1999; Edwards and Spurgeon, 1998; Alwi et al., 2011) is a nonlinear approach which is inherently robust against matched uncertainty. Due to the fact that actuator faults can be effectively modelled as matched uncertainty, the SMC approach (Edwards et al., 2012) is inherently advantageous from an FTC viewpoint because it "naturally" copes with actuator faults. However, in the case of a total actuator failure, redundancy and a mechanism to exploit advantageously the redundant actuators are required. The combination of SMC with CA provides a complete solution to handle both actuator faults and failures. To deal with actuator faults/failures, the most commonly employed strategy is to distribute the control effort equally amongst the actuators (Shtessel et al., 2002; Wells and Hess, 2003). However, to minimise the use of faulty actuators, or particularly reroute the control effort in the case of ineffective actuators, the schemes proposed by Alwi and Edwards (2008a; 2008b) use actuator effectiveness levels provided by a suitable fault detection and isolation scheme to achieve fault tolerance.

The FTC schemes of Alwi and Edwards (2008a; 2008b) as well as Hess and Wells (2003), due to the reaching phase, only guarantee tolerance against actuator faults/failures after the occurrence of the sliding mode (Utkin and Shi, 1996). To address this shortcoming, the idea of integral sliding mode (ISM) control was proposed by Utkin and Shi (1996) to eliminate the reaching phase, so that the sliding mode can be enforced throughout the closed-loop system response. Recently, the ideas of ISM control have been used in the framework of active FTC, for state feedback and output feedback systems (cf. Hamayun et al., 2012; 2013). In both the schemes of Hamayun et al. (2012; 2013), information about actuator effectiveness levels was explicitly used in the CA component.

The FTC scheme proposed in this paper considers the possibility of introducing fault tolerance without having information about actuator effectiveness levels or fault information from an FDI scheme. To provide the solution, the idea of direct control allocation is incorporated within the ISM FTC framework. The contribution of this paper as compared to the FTC schemes by Hamayun et al. (2012; 2013) is that the proposed scheme has the capability to deal with actuator faults/failures without any FDI scheme and is suitable for the case where fault information is not available to the controller. A detailed LMI based procedure is provided to synthesize the controller parameters, and a rigorous closed-loop stability analysis is carried out in the presence of unmatched uncertainty for a suitable set of actuator faults/failures. The proposed scheme is compared in simulation with the FTC scheme by Hamayun et al. (2012) by considering the same manoeuvre on a high fidelity nonlinear model of transport aircraft in the presence of wind, gusts and sensor noise.

The paper is organised as follows. Section 2 presents problem formulation and describes the model of an over-actuated system with actuator faults or failures. Section 3 describes the FTC scheme using integral sliding modes with direct control allocation. A detailed LMI based procedure is also provided to synthesize the controller parameters to ensure closed loop stability in the presence of unmatched uncertainty for a suitable set of actuator faults/failures. Sections 4 and 5 demonstrate how the proposed scheme's performance can be compared, in simulation, with the recently published active fault tolerant control scheme by Hamayun et al. (2012) by considering the same manoeuvre in the presence of wind, gusts and sensor noise, on a high fidelity nonlinear model of a transport aircraft. Finally, Section 6 concludes the paper by highlighting the main achievements of the work.

\section{Problem formulation}

Consider an over-actuated system with actuator faults or failures modelled as

$$
\dot{x}(t)=A x(t)+B W u(t)+D \xi(t, x),
$$

where $A \in \mathbb{R}^{n \times n}$ and $B \in \mathbb{R}^{n \times m}$ are the state and input distribution matrices, respectively. Here it is assumed that $B$ has full column rank. The term $W=\operatorname{diag}\left\{w_{1}, \ldots, w_{m}\right\}$ is a diagonal weighting matrix wherein the scalars $w_{1}, \ldots, w_{m}$ model the effectiveness/efficiency levels of the actuators. If the diagonal entry $w_{i}=1$, this indicates that the corresponding $i$-th actuator is healthy/fault-free, whereas if $1>w_{i}>0$, the $i$-th actuator is faulty. Finally, the value $w_{i}=0$ indicates that the corresponding actuator has failed. In this paper it is assumed that the actuator effectiveness level (i.e., the matrix $W$ ) is not available.

In Eqn. (1), the function $\xi(t, x)$ represents uncertainties/nonlinearities in the system. The structure of $D$ matrix is assumed to be such that $B^{T} D=0$, i.e., $D$ is in the null space of $B$. Assume that the input 


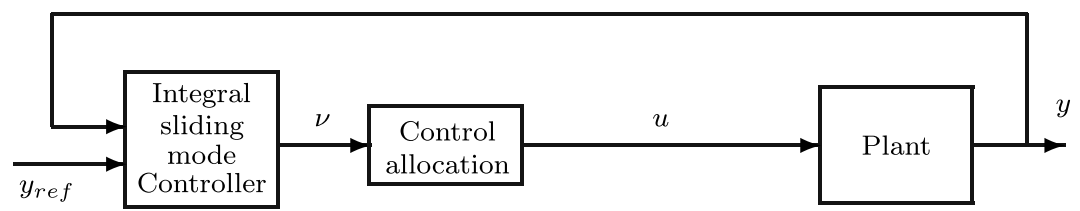

Fig. 1. FTC strategy with direct control allocation.

distribution matrix $B$ in (1) can be partitioned as

$$
B=\left[\frac{B_{1}}{B_{2}}\right] \text {, }
$$

where $B_{1} \in \mathbb{R}^{(n-l) \times m}$ and $B_{2} \in \mathbb{R}^{l \times m}$ is of rank $l<m$. It will be demonstrated later in the paper that the norm of $\left\|B_{1}\right\|$ plays a significant role in the closed-loop stability analysis. In this paper, as in the work of Alwi and Edwards (2008a), it is assumed that $\left\|B_{2}\right\|>\left\|B_{1}\right\|$, so that $B_{2}$ is more dominant in the delivery of the control effort to the system compared with $B_{1}$. Note that state permutation may be required to achieve the form in (2). It is also assumed without loss of generality that the states are scaled to ensure $B_{2} B_{2}^{T}=I_{l}$. This will help in simplifying the analysis and design in the sequel.

In order to track a reference demand, a tracking capability (Ogata, 2002) is included in the design. For this purpose, the states $x(t)$ in (1) are augmented with integral action states $x_{r}(t)$ creating the vector $x_{a}(t)=$ $\operatorname{col}\left[x_{r}(t), x(t)\right]$, where

$$
\dot{x}_{r}(t)=r(t)-C x(t) .
$$

In (3) the signal $r(t)$ is the reference command to be tracked, whereas $C \in \mathbb{R}^{l \times n}$ is the output distribution matrix which extracts the controlled outputs from the states. The overall augmented system becomes

$$
\dot{x}_{a}(t)=A_{a} x_{a}(t)+B_{a} W u(t)+B_{r} r(t)+D_{a} \xi(t, x),
$$

where the augmented matrices are

$$
\begin{array}{rlrl}
A_{a} & =\left[\begin{array}{cc}
0 & -C \\
0 & A
\end{array}\right], & B_{a}=\left[\begin{array}{c}
B_{1_{a}} \\
B_{2}
\end{array}\right], \\
B_{1_{a}}=\left[\begin{array}{c}
0 \\
B_{1}
\end{array}\right], & B_{r}=\left[\begin{array}{c}
I_{l} \\
0
\end{array}\right], \\
D_{a}=\left[\begin{array}{c}
0 \\
D
\end{array}\right] . &
\end{array}
$$

Note that, by definition, $B_{a}$ has full column rank (inherited from $B$ ). It is assumed that the function $\xi(t, x)$ satisfies the relation $\xi(t, x)=\phi(t, x) x$, where $\|\phi(t, x)\|$ is bounded. In the next section, an integral sliding mode FTC scheme with direct CA will be proposed for the system in (4).

\section{Integral sliding mode FTC scheme with direct control allocation}

In the FTC scheme which will be proposed, the fault information (i.e., the matrix $W$ ) is not required for the control allocation scheme and hence the scheme can be regarded as "passive" (Verhaegen et al., 2010). The proposed strategy "distributes" the control effort among all the actuators and constitutes a passive fault tolerant control scheme. The ISM FTC scheme, incorporating direct CA, is shown in Fig. 1,

In this paper, a virtual control input (Harkegard and Glad, 2005), $\nu(t) \in \mathbb{R}^{l}$, is defined as

$$
\nu(t)=B_{2} u(t),
$$

where $B_{2}$ is taken from (5). Here the physical control law $u(t)$ is chosen as

$$
u(t)=B_{2}^{T} \nu(t) .
$$

Using the fact that $B_{2} B_{2}^{T}=I_{l}, B_{2}^{T}$ is a right pseudo-inverse of $B_{2}$, and hence the choice of the control law in (7) satisfies (6). In the sequel, a framework for the design of the virtual control law $\nu(t)$ will be formulated. Substituting (7) into (4) yields

$$
\begin{aligned}
\dot{x}_{a}(t)= & A_{a} x_{a}(t)+\underbrace{\left[\begin{array}{c}
B_{1_{a}} W B_{2}^{T} \\
B_{2} W B_{2}^{T}
\end{array}\right]}_{B_{w_{a}}} \nu(t) \\
& +B_{r} r(t)+D_{a} \xi(\cdot) .
\end{aligned}
$$

The so-called ISM approach (Utkin and Shi, 1996) will be used to design the virtual controller $\nu(t)$ in (8), based on the nominal fault free system (i.e., when $W=$ $\left.I_{m}\right)$. Substituting $W=I_{m}$ in 8 yields

$$
\begin{aligned}
\dot{x}_{a}(t)= & A_{a} x_{a}(t)+\underbrace{\left[\begin{array}{c}
B_{1_{a}} B_{2}^{T} \\
I_{l}
\end{array}\right]}_{B_{\nu_{a}}} \nu(t) \\
& +B_{r} r(t)+D_{a} \xi(\cdot),
\end{aligned}
$$

where it is assumed that the pair $\left(A_{a}, B_{\nu_{a}}\right)$ is (preferably) controllable but at least stabilizable. Note that

$$
\left\|B_{1 a} B_{2}^{T}\right\| \leq\left\|B_{1 a}\right\|\left\|B_{2}^{T}\right\| \leq\left\|B_{1 a}\right\| \leq\left\|B_{1}\right\|
$$

because $\left\|B_{2}^{T}\right\|=1$ as $B_{2} B_{2}^{T}=I$, and the last inequality follows from the definition in (5). Consequently, the 
control contribution in the first $n$ channels of (9) is still small. To design $\nu(t)$ based on an ISM methodology, an integral switching function which eliminates the reaching phase and aims to retain the nominal closed-loop performance is defined as

$$
\begin{aligned}
& \sigma\left(x_{a}, t\right) \\
& =G_{a} x_{a}(t)-G_{a} x_{a}\left(t_{0}\right) \\
& \quad-G_{a} \int_{t_{0}}^{t}\left(\left(A_{a}-B_{\nu_{a}} F\right) x_{a}(\tau)+B_{r} r(\tau)\right) \mathrm{d} \tau,
\end{aligned}
$$

where the matrix $G_{a} \in \mathbb{R}^{l \times(n+l)}$ is design freedom. In (10), the matrix $F \in \mathbb{R}^{l \times(n+l)}$ is a state feedback gain chosen to make the matrix $\left(A_{a}-B_{\nu_{a}} F\right)$ Hurwitz. The associated sliding motion occurs on the surface $\mathcal{S}=$ $\left\{x_{a} \in \mathbb{R}^{n+l}: \sigma\left(x_{a}, t\right)=0\right\}$. The choice of $G_{a}$ suggested by Hamayun et al. (2012), which will also be employed here, is

$$
G_{a}:=B_{2}\left(B_{a}^{T} B_{a}\right)^{-1} B_{a}^{T},
$$

which is a left pseudo-inverse of $B_{\nu_{a}}$, i.e., $G_{a} B_{\nu_{a}}=I$. The inverse in (11) exists because the control distribution matrix $B_{a}$ is assumed to have full column rank. The choice of $G_{a}$ in (11) brings some simplifying properties to the closed-loop analysis, which are explained later. The sliding motion associated with the surface in (10), in the presence of faults or failures, will now be analysed.

The time derivative of (10) along the trajectories of the differential equation $(8)$ is given by

$$
\dot{\sigma}_{a}\left(x_{a}, t\right)=G_{a} B_{w_{a}} \nu(t)+G_{a} B_{\nu_{a}} F x_{a}(t) .
$$

Note that (12) does not depend on the reference signal $r(t)$ because

$$
\begin{aligned}
G_{a} B_{r} & =B_{2}\left(B_{a}^{T} B_{a}\right)^{-1} B_{a}^{T} B_{r} \\
& =B_{2}\left(B_{a}^{T} B_{a}\right)^{-1}\left[\begin{array}{ll}
0 & B^{T}
\end{array}\right]\left[\begin{array}{c}
I_{l} \\
0
\end{array}\right]=0
\end{aligned}
$$

Also note that (12) is independent of the uncertainty $\xi(t, x)$ because

$$
G_{a} D_{a}=B_{2}\left(B_{a}^{T} B_{a}\right)^{-1} \underbrace{B_{a}^{T} D_{a}}_{0}=0
$$

by assumption. In order to obtain an expression for the closed-loop motion during sliding, an equivalent control approach (Utkin et al., 1999) will be adopted.

The equivalent control (Utkin et al., 1999) which maintains the motion on the surface in (10) is obtained by equating $\dot{\sigma}_{a}=0$ in (12) to obtain

$$
\nu_{\mathrm{eq}}(t)=-\left(G_{a} B_{w_{a}}\right)^{-1} G_{a} B_{\nu_{a}} F x_{a}(t) .
$$

Note the expression in $(13)$ is not the virtual control signal used in (7) to obtain the physical control signal, but it is purely an abstraction used to obtain an expression for the sliding motion (Utkin et al., 1999). For the existence of such a control, $G_{a} B_{w_{a}}$ needs to be nonsingular, which will be discussed later in Assumption 1. It is easy to verify that, with the choice of $G_{a}$ in (11),

$$
G_{a} B_{w_{a}}=B_{2}\left(B_{a}^{T} B_{a}\right)^{-1}\left(B_{a}^{T} B_{a}\right) W B_{2}^{T}=B_{2} W B_{2}^{T} .
$$

Therefore, the expression for the equivalent control in 13 can be simplified to

$$
\nu_{\mathrm{eq}}(t)=-\left(B_{2} W B_{2}^{T}\right)^{-1} F x_{a}(t) .
$$

Assumption 1. It is assumed that $W$ belongs to the set $\mathcal{W}=\left\{\left(w_{1}, \ldots, w_{m}\right): \lambda_{\min }\left(B_{2} W B_{2}^{T}\right)>\lambda_{o}\right\}$, where $\lambda_{o}$ is a positive design scalar.

Remark 1. As $l<m$, it is possible that $\operatorname{det}\left(B_{2} W B_{2}^{T}\right) \neq$ 0 even if up to $m-l$ of the entries $w_{i}$ are zero in the matrix $W$. This means that up to $m-l$ actuators can be subjected to total failure and yet $\operatorname{det}\left(B_{2} W B_{2}^{T}\right) \neq 0$. However, if more than $m-l$ entries become zero, then $\operatorname{rank}(W)<l$ and $\operatorname{det}\left(B_{2} W B_{2}^{T}\right)=0$. The set $\mathcal{W}$ consists of the faults or failures which the scheme proposed in this paper can cope with.

The dynamics associated with the sliding surface $\mathcal{S}$ can be obtained by substituting the equivalent control from (14) into 8), which yields

$$
\begin{aligned}
\dot{x}_{a}(t)= & A_{a} x_{a}(t)-B_{w_{a}}\left(B_{2} W B_{2}^{T}\right)^{-1} F x_{a}(t) \\
& +B_{r} r(t)+D_{a} \xi(t, x) .
\end{aligned}
$$

Adding and subtracting the term $B_{\nu_{a}} F x_{a}(t)$ to (15) and rearranging mean that (15) can also be written as

$$
\begin{aligned}
\dot{x}_{a}(t)= & \left(A_{a}-B_{\nu_{a}} F\right) x_{a}(t)+B_{m} F x_{a}(t) \\
& +B_{r} r(t)+D_{a} \xi(\cdot),
\end{aligned}
$$

where

$$
B_{m}:=\left[\begin{array}{c}
B_{1_{a}} B_{2}^{T}-B_{1_{a}} W B_{2}^{T}\left(B_{2} W B_{2}^{T}\right)^{-1} \\
0
\end{array}\right] .
$$

Note that in Eqn. (16), since $r(t)$ is a bounded signal, the closed-loop stability of the sliding motion only depends on the first two and the last terms, which can be expressed in the form

$$
\dot{x}_{a}(t)=\underbrace{\left(A_{a}-B_{\nu_{a}} F\right)}_{\tilde{A}} x_{a}(t)+\tilde{B}_{a} \overbrace{\Delta_{a f}(t) \underbrace{F_{a} x_{a}(t)}_{\tilde{y}}}^{\tilde{u}},
$$

where

$$
\begin{aligned}
\tilde{B}_{a} & =\left[\begin{array}{cc}
\tilde{B} & D_{a}
\end{array}\right], & \tilde{B} & :=\left[\begin{array}{c}
I_{n} \\
0
\end{array}\right] \\
\Delta_{a f} & =\left[\begin{array}{cc}
\Delta_{f} & 0 \\
0 & \tilde{\phi}(t, x)
\end{array}\right], & F_{a} & =\left[\begin{array}{c}
F \\
I
\end{array}\right],
\end{aligned}
$$


and

$$
\tilde{\phi}(t, x)=\left[\begin{array}{ll}
0 & \phi(t, x)
\end{array}\right] .
$$

The uncertainty fault/failure dependent term is

$$
\Delta_{f}(t):=B_{1_{a}} B_{2}^{T}-B_{1_{a}} W B_{2}^{T}\left(B_{2} W B_{2}^{T}\right)^{-1} .
$$

Note that since $W$ is unknown, and $\tilde{\phi}(t, x)$ is assumed to be bounded, the expression $\Delta_{a f}(t)$ above is treated as an uncertainty. To facilitate the closed-loop stability analysis, assume

$$
\left\|\Delta_{a f}(t)\right\|<\gamma_{1}, \quad \forall W \in \mathcal{W},
$$

where $\gamma_{1}$ is a positive scalar. Such a bound is guaranteed to exist since $\|W\|<1$ and $\left\|B_{2} W B_{2}^{T}\right\|<1 / \lambda_{o}$. Define

$$
\gamma_{2}=\|\tilde{G}(s)\|_{\infty},
$$

where the transfer function matrix is

$$
\tilde{G}(s):=F_{a}(s I-\tilde{A})^{-1} \tilde{B}_{a} .
$$

Note that, by the design of $F$, the matrix $\tilde{A}=A_{a}-B_{a} F$ is stable, and so the $H_{\infty}$-norm of $\gamma_{2}$ is well defined and finite.

Proposition 1. For any combination of faults or failures belonging to the set $\mathcal{W}$, the sliding motion in (18) will be stable if

$$
\gamma_{2} \gamma_{1}<1
$$

where $\gamma_{1}$ and $\gamma_{2}$ are defined in (21) and (22), respectively.

Proof. The structure of differential equation (18) can be considered the interconnection of the gain $\tilde{G}(s)$ (in the feedforward path) with the uncertain term $\Delta_{a f}(t)$ (in the feedback loop) of a closed-loop system. Using Eqns. (21) to (23), and from the small gain theorem (Khalil, 1992), if

$$
\|\tilde{G}(s)\|_{\infty}\left\|\Delta_{a f}(t)\right\|<1,
$$

then the closed-loop stability of $(18)$ is ensured. The condition in (25) is exactly the stability condition in the statement of the proposition and the proof is complete.

In a sliding mode framework, the control law should be designed to satisfy the so-called reachability condition (Edwards and Spurgeon, 1998), which is a sufficient condition to guarantee that sliding will be enforced and maintained.

Proposition 2. The virtual control based on the integral sliding mode control law

$$
\nu(t)=\nu_{l}(t)+\nu_{n}(t)
$$

where

$$
\begin{aligned}
\nu_{l}(t) & :=-F x_{a}(t), \\
\nu_{n}(t) & :=-\rho(t) \frac{\sigma_{a}\left(x_{a}, t\right)}{\left\|\sigma_{a}\left(x_{a}, t\right)\right\|} \text { for } \sigma_{a}\left(x_{a}, t\right) \neq 0,
\end{aligned}
$$

will maintain sliding during faults or failures belonging to the set $\mathcal{W}$ if the modulation gain $\rho(t)$ in (28) is selected as

$$
\rho(t)>\frac{2\left\|\nu_{l}(t)\right\|+\eta}{\lambda_{o}},
$$

where $\eta$ is a positive design scalar.

Proof. From substituting Eqn. (26) into (12) and using the fact $G_{a} B_{w_{a}}=B_{2} W B_{2}^{T}$ and $G_{a} B_{\nu_{a}}=I$, it follows that

$$
\begin{aligned}
\dot{\sigma}_{a}(\cdot) & =\left(B_{2} W B_{2}^{T}\right)\left(\nu_{l}(t)+\nu_{n}(t)\right)+F x_{a}(t) \\
& =\left(B_{2} W B_{2}^{T}\right) \nu_{n}(t)+\left(B_{2} W B_{2}^{T}\right) \nu_{l}(t)+F x_{a}(t) .
\end{aligned}
$$

Consider a positive definite candidate Lyapunov function $V=\frac{1}{2} \sigma_{a}^{T} \sigma_{a}$. Taking the time derivative along the system trajectories gives

$$
\begin{aligned}
\dot{V}= & \sigma_{a}^{T}\left(\left(B_{2} W B_{2}^{T}\right) \nu_{n}(t)+\left(B_{2} W B_{2}^{T}\right) \nu_{l}(t)+F x_{a}(t)\right) \\
\leq & -\lambda_{\min }\left(B_{2} W B_{2}^{T}\right) \rho\left\|\sigma_{a}\right\|+\left\|\sigma_{a}\right\|\left\|B_{2} W B_{2}^{T}\right\|\left\|\nu_{l}\right\| \\
& +\left\|\sigma_{a}\right\|\left\|F x_{a}(t)\right\| \\
\leq & -\lambda_{o} \rho\left\|\sigma_{a}\right\|+\left\|\sigma_{a}\right\|\left\|\nu_{l}\right\|+\left\|\sigma_{a}\right\|\left\|\nu_{l}\right\|
\end{aligned}
$$

since $\left\|B_{2} W B_{2}^{T}\right\|<1$.

By using a value of $\rho(t)$ satisfying (29), the inequality (31) can be written as

$$
\dot{V} \leq-\eta\left\|\sigma_{a}\right\|=-\sqrt{2} \eta V^{\frac{1}{2}},
$$

which is a standard reachability condition (Edwards and Spurgeon, 1998), and the proof is complete.

Finally, substituting the integral sliding mode control law given in (26)-(28) into (77) yields the physical control law

$$
u(t)=B_{2}^{T}\left(-F x_{a}(t)-\rho \frac{\sigma_{a}\left(x_{a}, t\right)}{\left\|\sigma_{a}\left(x_{a}, t\right)\right\|}\right) .
$$

It is clear that the physical control law $u(t)$ in $(33)$ has no information about the actuator effectiveness matrix $W$ and will distribute the control effort amongst all the actuators.

3.1. Design of feedback gain $\boldsymbol{F}$. The feedback gain $F$ associated with the physical control law $u(t)$ in (33) can be designed by using the LMI optimization method proposed by Hamayun et al. (2012) (which is also explained in this paper for completeness) such that the small gain stability condition given in Proposition 1 is satisfied. To have a finite value of $\gamma_{2}$, the matrix $\tilde{A}=$ $A_{a}-B_{a} F$ in (23) should be stable. The feedback gain $F$ is designed to meet the nominal performance requirements (when $W=I_{m}$ ) whilst also satisfying the stability condition in Proposition 1. For nominal performance specification, an LQR formulation is adopted, which 
can be posed as an LMI optimization as follows (Boyd et al., 1994): Minimize trace $\left(X^{-1}\right)$ subject to

$$
\begin{gathered}
{\left[\begin{array}{c}
A_{a} X+X A_{a}^{T}-B_{\nu_{a}} Y-Y^{T} B_{\nu_{a}}^{T} \\
\hat{Q} X-\hat{R} Y \\
(\hat{Q} X-\hat{R} Y)^{T} \\
-I
\end{array}\right]<0,} \\
X>0,
\end{gathered}
$$

where $Y:=F X$ and the symmetric matrix $X \in$ $\mathbb{R}^{(n+l) \times(n+l)}$. We have

$$
\hat{Q}:=\left[\begin{array}{ll}
Q^{1 / 2} & 0
\end{array}\right]^{T}, \quad \hat{R}:=\left[\begin{array}{ll}
0 & R^{1 / 2}
\end{array}\right]^{T} .
$$

To encapsulate the small gain stability condition (24), the bounded real lemma (Boyd et al., 1994) is used:

$$
\left[\begin{array}{ccc}
A_{a} X+X A_{a}^{T}-B_{\nu_{a}} Y-Y^{T} B_{\nu_{a}}^{T} & \tilde{B}_{a} & Y^{T} \\
\tilde{B}_{a}^{T} & -\gamma^{2} I & 0 \\
Y & 0 & -I
\end{array}\right]<0
$$

where $\tilde{B}_{a}$ is defined in 19 . The scalar gain $\gamma$ may be viewed as a tuning parameter.

The overall optimization process is as follows: Minimize trace $(Z)$ subject to

$$
\left[\begin{array}{cc}
-Z & I_{n} \\
I_{n} & -X
\end{array}\right]<0
$$

together with (34), 35) and (36). The slack variable $Z$ satisfies $Z>X^{-1}$, and therefore $\operatorname{trace}(Z) \geq \operatorname{trace}\left(X^{-1}\right)$. Finally, the feedback gain $F$ can be recovered as $F=$ $Y X^{-1}$.

\section{Simulations}

A high-fidelity non-linear benchmark model of a large passenger aircraft based on the FTLAB747 v6.1/v6.5 software (Marcos and Balas, 2003) is used to test the effectiveness of the proposed FTC scheme. This software represents a "real world" model of the large body B747-100/200 aircraft.

The feedback gain $F$ was designed based on a linearization of the model around an operating condition of a straight and level flight at 263,000 kg, $92.6 \mathrm{~m} / \mathrm{s}$ true airspeed, and an altitude of $600 \mathrm{~m}$ based on $25.6 \%$ of maximum thrust, and at a $20 \mathrm{deg}$ flap position. In the simulations, an up-and-away flight manoeuvre is considered. For longitudinal control only the states $x=$ $\left[\theta, \alpha, V_{\text {tas }}, q\right]^{T}$ are examined, where $\theta$ is the pitch angle $(\mathrm{rad}), \alpha$ is the angle of attack ( $\mathrm{rad}), V_{\text {tas }}$ is the true airspeed $(\mathrm{m} / \mathrm{sec})$, and $q$ is the pitch rate $(\mathrm{rad} / \mathrm{sec})$. The available longitudinal control surfaces are $\delta_{\text {long }}=\left[\delta_{e}, \delta_{s}, \delta_{\text {epr }}\right]^{T}$ and represent aggregated elevator deflection (rad), horizontal stabilizer deflection (rad), and aggregated longitudinal EPR (i.e., the four engine pressure ratios (EPRs) are aggregated to produce one control input). The state space model obtained at the operating condition of a straight and level flight is

$$
\begin{gathered}
A=\left[\begin{array}{rrrr}
0 & 0 & 0 & 1 \\
0 & -0.6284 & -0.0021 & 1.0064 \\
-9.8046 & 1.7171 & -0.0166 & 0 \\
0 & -0.5831 & 0.0004 & -0.5137
\end{array}\right], \\
B=\left[\begin{array}{rrr}
0 & 0 & 0 \\
-0.0352 & -0.0819 & -0.0084 \\
\hline & & \\
0 & -0.1756 & 5.7072 \\
-0.6228 & -1.3578 & 0.0600
\end{array}\right],
\end{gathered}
$$

where the input matrix $B$ is partitioned according to (2).

For the up-and-away manoeuvre it is required to track the flight path angle (FPA) $\gamma$ (where $\gamma=\theta-\alpha$ ) and $V_{\text {tas. }}$. Therefore, the controlled output matrix is

$$
C=\left[\begin{array}{cccc}
1 & -1 & 0 & 0 \\
0 & 0 & 1 & 0
\end{array}\right]
$$

To design the state feedback gain $F$, the LMI approach given in Section 3.1 was used, in which the design matrices were $Q=\operatorname{diag}(0.95,0.004,0.01,2,0.1,5)$ and $R=\operatorname{diag}(4,8)$. With the choice of $\gamma=7$ in (36), the feedback gain $F$ obtained is

$$
\begin{aligned}
& F=\left[\begin{array}{rrrr}
-0.1714 & -0.0296 & -0.7450 & -0.8077 \\
-0.3226 & 0.0079 & 2.0833 & 1.2277
\end{array}\right. \\
& 0.9828-0.2921] \\
& -0.1549 \quad 1.8162] \text {. }
\end{aligned}
$$

In this example the elevator is the primary control surface for FPA tracking while the horizontal stabilizer is used as a redundant control surface. However, for $V_{\text {tas }}$ tracking, aggregated EPR is the only available actuator (and has no redundancy). Based on the assumption that the engines are fault free, we used a search parameter value of $\lambda_{0}=$ 0.0826 and a suitable upper bound in Eqn. (21) was set as $\gamma_{1}=0.01$. With the feedback gain $F$ given above, it can be verified that $\gamma_{2}=75.3013$ in (22), which fulfils the stability requirement of Proposition 1.

\section{Nonlinear simulation results}

In the simulations, a series of 3 deg FPA pulses, together with a change in $V_{\text {tas }}$ of $10 \mathrm{~m} / \mathrm{sec}$, are used to increase the altitude and the speed of the aircraft. In the simulations the discontinuity associated with the nonlinear controller term in (28) was smoothed by the sigmoidal approximation $\sigma_{a} /\left(\left\|\sigma_{a}\right\|+\delta\right)$ (Edwards and Spurgeon, 1998), where $\delta$ is chosen small (here $\delta=0.05$ ). (In so doing, a pseudo-sliding motion takes place in which the system trajectories move in the vicinity of the sliding surface.) To enforce sliding during faults/failures belonging to the 

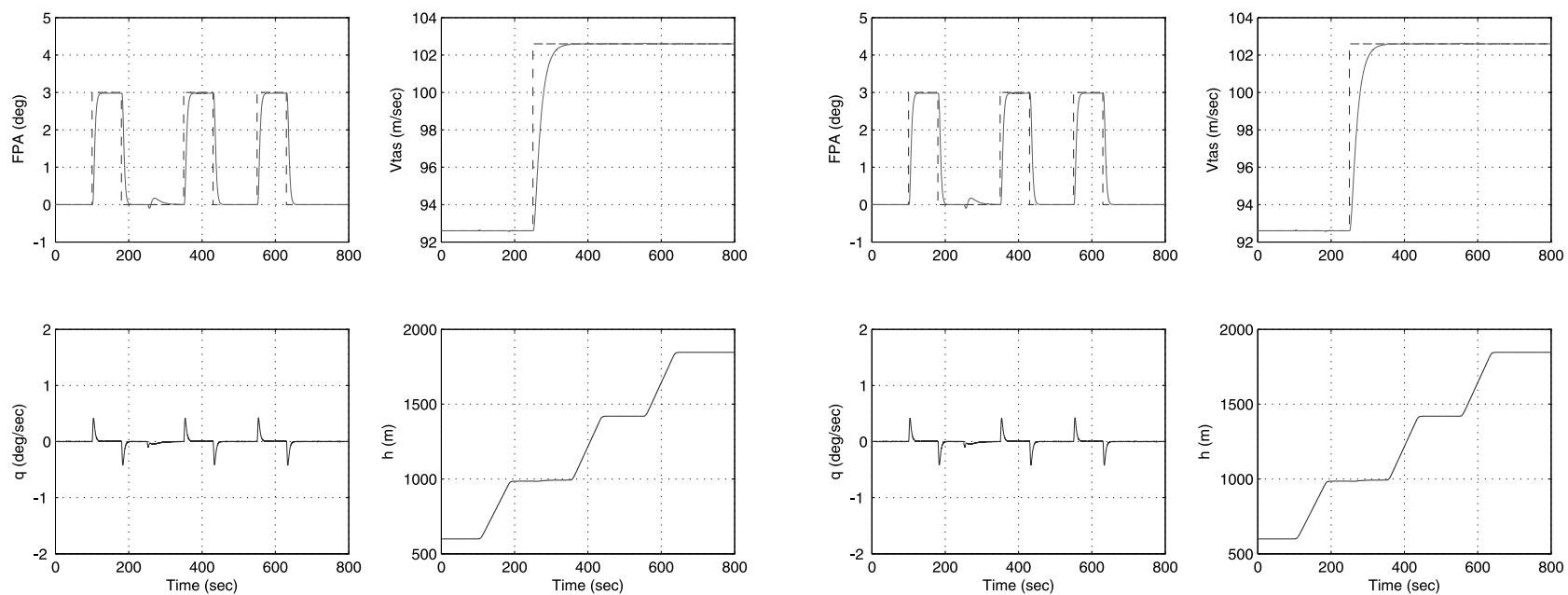

Fig. 2. Nominal fault free performance: system states based on a direct control allocation scheme.
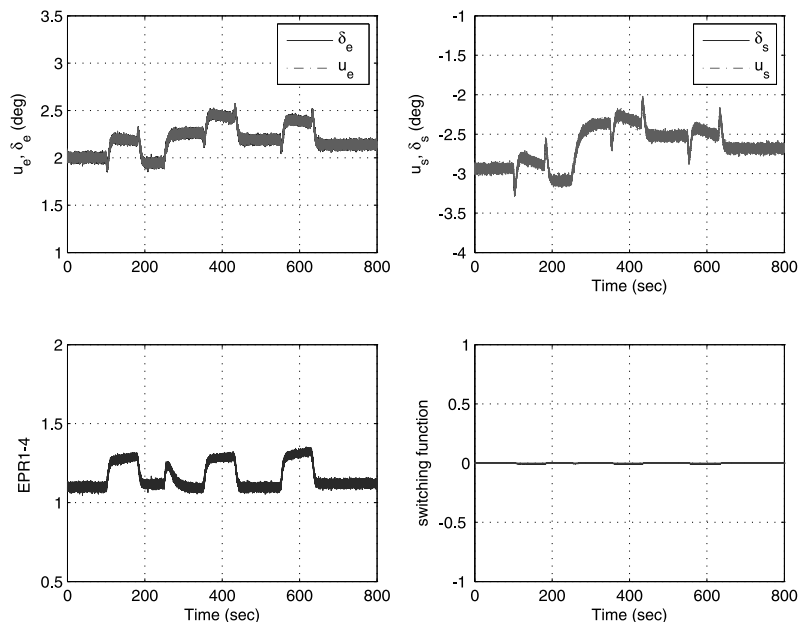

Fig. 3. Nominal fault free performance: actuator deflections based on a direct control allocation scheme.

set $\mathcal{W}$, the design scalars in 29) are chosen as $\eta=1$ and $\lambda_{0}=0.0826$, which satisfies the requirements of Proposition 2.

The scheme proposed in this paper is also compared in simulations with the recent FTC scheme by Hamayun et al. (2012). Note that those authors tested the online control allocation FTC scheme on a linear model of the lateral dynamics of a passenger aircraft; however, in this paper, the efficacy of both schemes will be compared based on the longitudinal dynamics of a high-fidelity nonlinear aircraft model.

Details of the longitudinal controller design based on the approach of Hamayun et al. (2012) are given by Hamayun (2013, Chapter 5). All the simulations in this paper are obtained in the presence of wind and gust
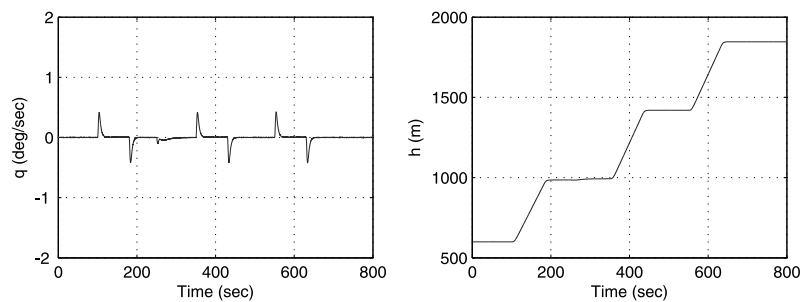

Fig. 4. Elevator with a partial to complete failure: system states based on an on-line control allocation scheme.
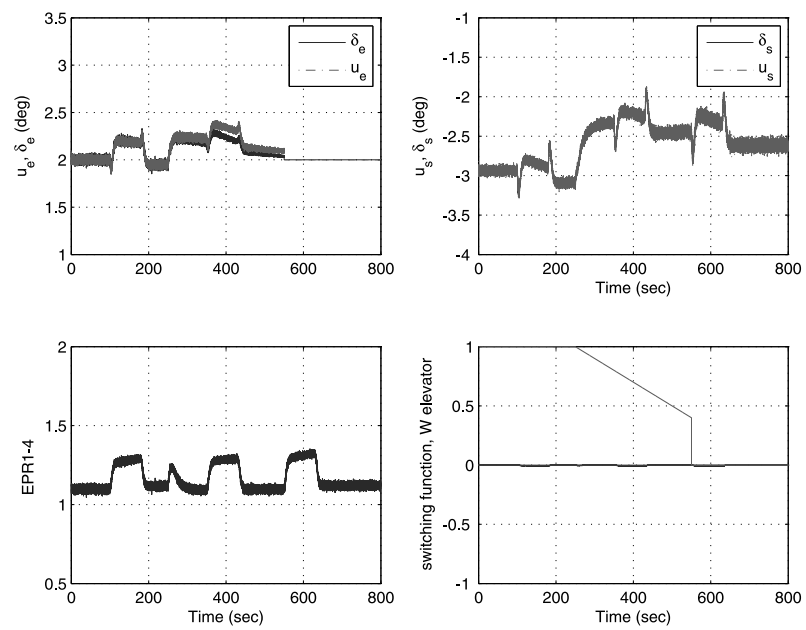

Fig. 5. Elevator with a partial to complete failure: actuator deflections based on an on-line control allocation scheme.

models and sensor noise. The wind model generates wind velocities $\left(u_{\text {wind }}=-11, v_{\text {wind }}=-12\right.$ and $w_{\text {wind }}=$ 0 ) along the positive axis of the earth reference frame, whereas the Dryden spectra are used in the gust model (Marcos and Balas, 2003). Both the models are embedded in the FTLAB747 v6.1/v6.5 software. The sensor noise which appears in the measured states $\left(\theta, \alpha, V_{\text {tas }}, q\right)^{T}$ and is based on a Gaussian distribution of zero mean and variance $\left(3 e^{-8}, 1 e^{-2}, 3 e^{-6}, 3 e^{-8}\right)$.

In Figs. 2 and 3 the nominal fault free performance using the approach proposed in this paper is shown. The control signals $u(t)$ are also plotted, together with the actual actuator deflections for comparison with the online CA scheme proposed by Hamayun et al. (2012). The tracking performance of the commands $\gamma$ and 

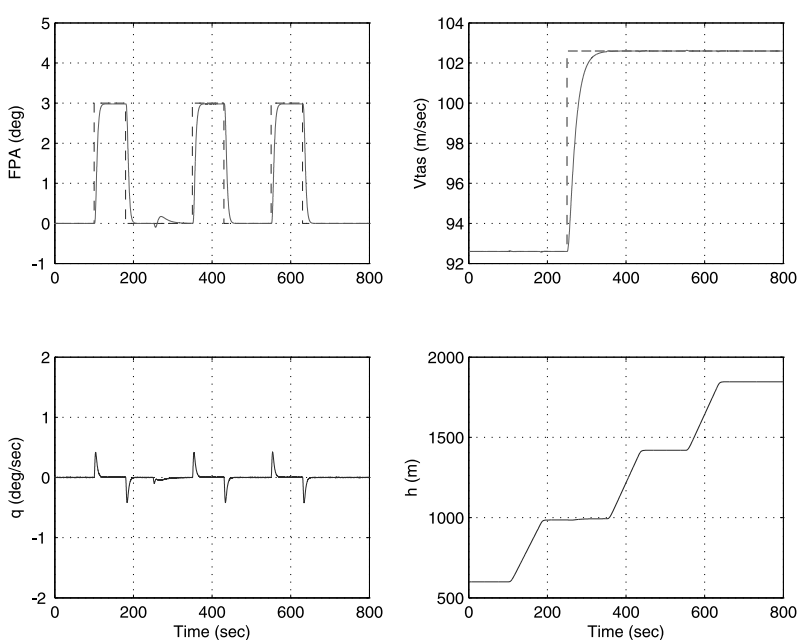

Fig. 6. Elevator with a partial to complete failure: system states based on a direct control allocation scheme.
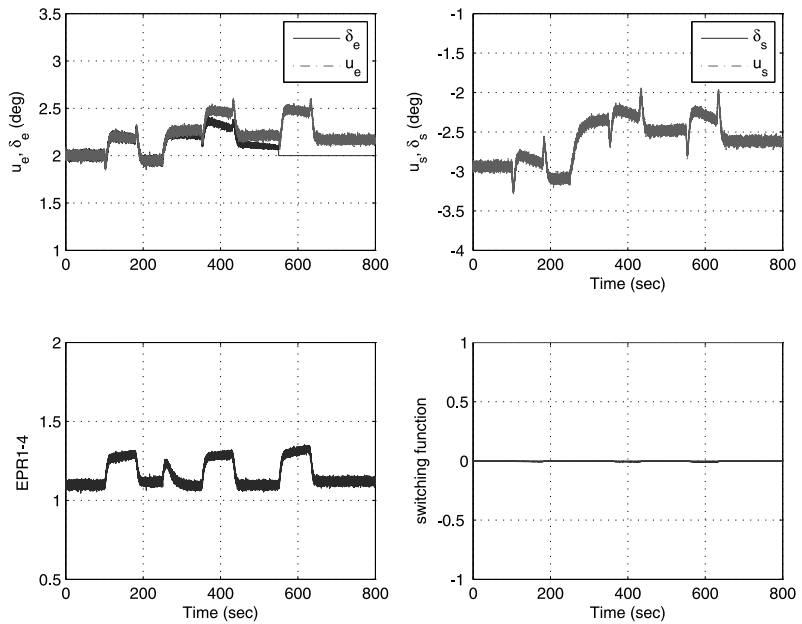

Fig. 7. Elevator with a partial to complete failure: actuator deflections based on a direct control allocation scheme.

$V_{\text {tas }}$ is promising. In Figs. 5 and 7 a fault scenario is demonstrated whereby the elevator's effectiveness decreases from $100 \%$ to $40 \%$ during $250-550 \mathrm{~s}$; after that, the elevator completely fails. It is clear from Figs. 4 and 6 that good tracking performance (close to nominal) is still achieved with both the scheme proposed by Hamayun et al. (2012) and the one given in this paper.

For on-line CA, it is clear from Fig. 5that the scheme of Hamayun et al. (2012) stops sending control signals to the elevator after it failed completely at $550 \mathrm{~s}$ due to the availability of information about the fault ( $W$ matrix) to the controller. The scheme proposed in this paper distributes control effort amongst all the actuators (Fig. 7) despite a fault/failure to the elevator as fault information is not available to the controller.

Figures 9 and 11 present the case when the elevator
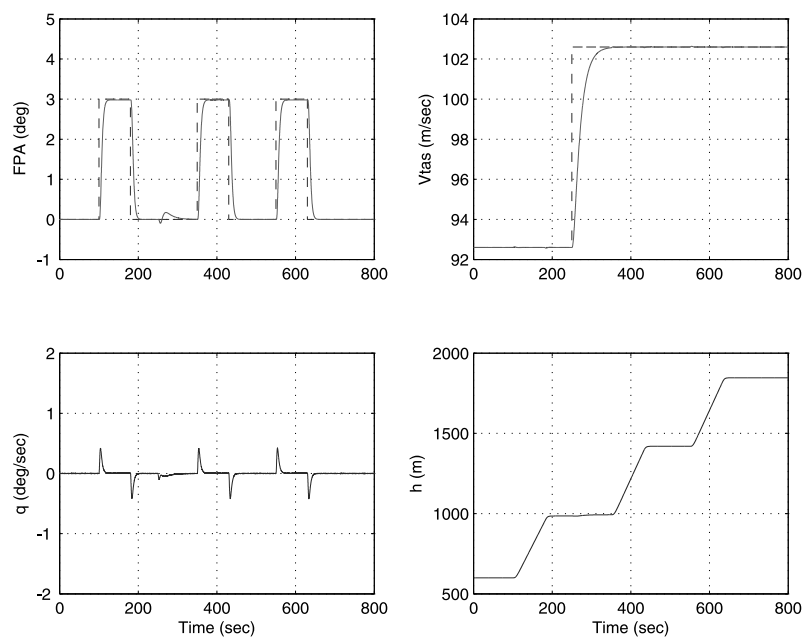

Fig. 8. Elevator jam: system states based on an on-line control allocation scheme.
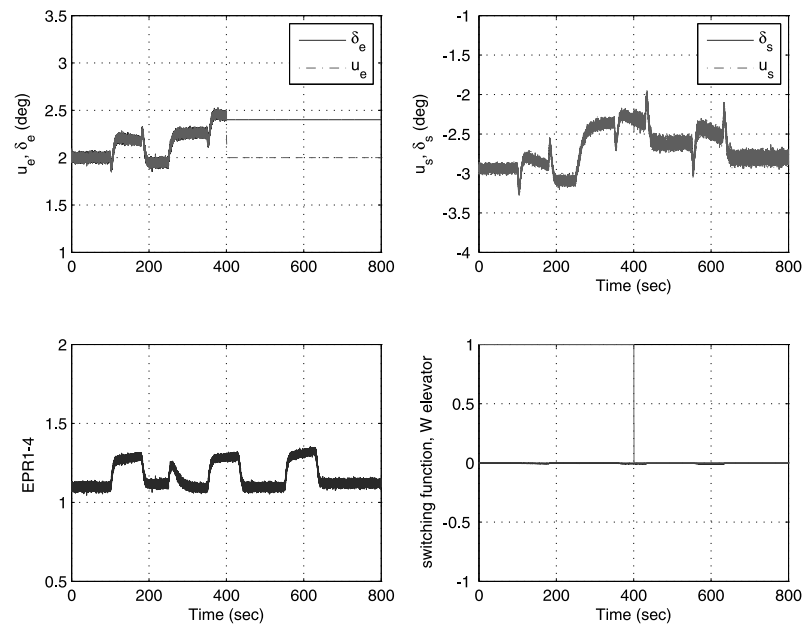

Fig. 9. Elevator jam: actuator deflections based on an on-line control allocation scheme.

jams at some offset position for both the online and direct CA schemes. However, due to the availability of the redundant control surface (the horizontal stabilizer), both the schemes can cope with this failure and still maintain the sliding motion as seen in Figs. 8 and 10, No apparent degradation in performance shows the effectiveness of both the schemes despite the severe failure condition. Based on the comparison between direct CA with on-line $\mathrm{CA}$, it can be seen that, despite the absence of FDI information (matrix $W$ ), the results for the proposed direct CA scheme are comparable to the online CA scheme of Hamayun et al. (2012). The only limitation of not knowing the actuator effectiveness level (matrix $W$ ) as compared to Hamayun et al. (2012) is that a slightly more limited set of faults/failures given in Assumption 1 must be assumed. 

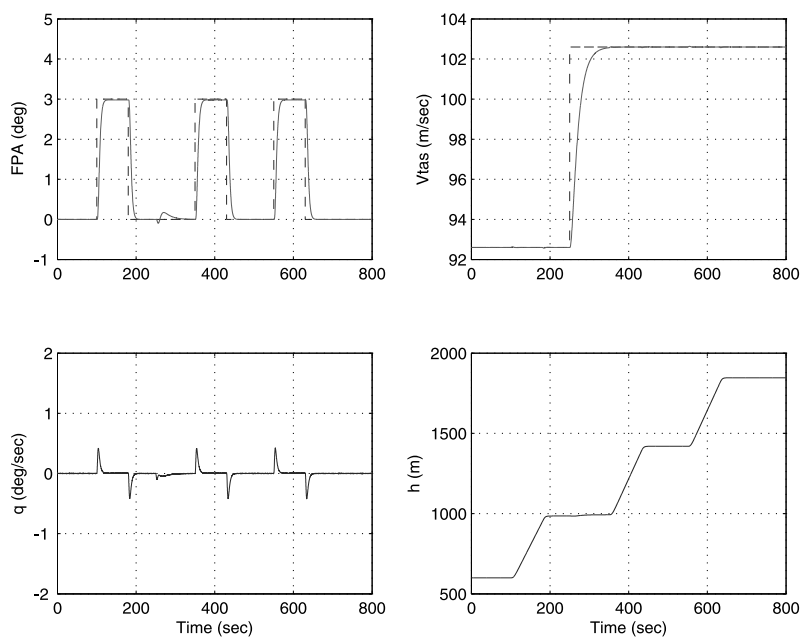

Fig. 10. Elevator jam: system states based on a direct control allocation scheme.
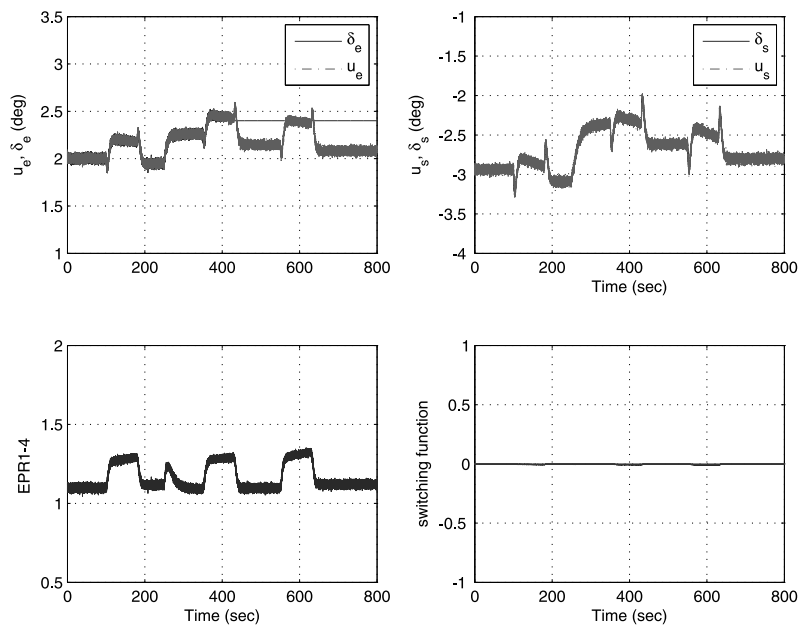

Fig. 11. Elevator jam: actuator deflections based on a direct control allocation scheme.

\section{Conclusion}

In this paper, a new passive FTC scheme was proposed which has the potential to provide fault tolerance against actuator faults/failures without requiring any information from an FDI scheme. The inclusion of direct control allocation allows the single controller to deal with a range of actuator faults/failures without any fault information. The direct control allocation structure maintains an acceptable level of closed-loop performance both nominally and in fault/failure situations by distributing the control effort computed by a virtual control law amongst the actuators.

A rigorous closed-loop stability analysis has been carried out in the presence of unmatched uncertainty and demonstrate the class of faults/failures which can be dealt with using the proposed scheme, without any performance degradation. A range of actuator fault/failure scenarios were considered in simulations, in the presence of wind, gusts and sensor noise, for validation purposes, on a high fidelity nonlinear aircraft model, and the results obtained show the effectiveness of the proposed scheme. The controller is also compared with an existing scheme, which does require fault information, and comparable results were obtained.

\section{Acknowledgment}

This paper was partially funded by the Deanship of Scientific Research (DSR), King Abdulaziz University, Jeddah, under the grant no. Gr/33/5. The first and the last author, therefore, acknowledge with thanks the DSR financial support.

\section{References}

Alwi, H. and Edwards, C. (2008a). Fault tolerant control using sliding modes with on-line control allocation, Automatica 44(7): 1859-1866.

Alwi, H. and Edwards, C. (2008b). Fault tolerant sliding mode control design with piloted simulator evaluation, Journal of Guidance, Control, and Dynamics 31(5): 1186-1201.

Alwi, H., Edwards, C. and Tan, C. (2011). Fault Detection and Fault Tolerant Control Using Sliding Modes, Advances in Industrial Control, Springer-Verlag, London.

Bošković, J. and Mehra, R. (2002). Control allocation in overactuated aircraft under position and rate limiting, American Control Conference, Anchorage, AK, USA, pp. 791-796.

Boyd, S., Ghaoui, L., Feron, E. and Balakrishnan, V. (1994). Linear Matrix Inequalities in System and Control Theory, SIAM, Philadelphia, PA.

Castaldi, P., Mimmo, N. and Simani, S. (2011). Fault tolerant control schemes for nonlinear models of aircraft and spacecraft systems, 18th IFAC World Congress, Milan, Italy, pp. 13705-13710.

Castaldi, P., Mimmo, N. and Simani, S. (2014). Differential geometry based active fault tolerant control for aircraft, Control Engineering Practice 32: 227-235, DOI: 10.1016/j.conengprac.2013.12.011i.

Davidson, J., Lallman, F. and Bundick, W. (2001). Real-time adaptive control allocation applied to a high performance aircraft, 5th SIAM Conference on Control and Its Application, Hampton, VA, USA, pp. 1-1.

Ducard, G.J.J. (2009). Fault-tolerant Flight Control and Guidance Systems: Practical Methods for Small Unmanned Aerial Vehicles, Advances in Industrial Control, Springer-Verlag, London.

Edwards, C., Alwi, H. and Tan, C.P. (2012). Sliding mode methods for fault detection and fault tolerant control with application to aerospace systems, International Journal of Applied Mathematics and Computer Science 22(1): 109-124, DOI: 10.2478/v10006-012-0008-7. 
Edwards, C., Lombaerts, T. and Smaili, H. (2010). Fault Tolerant Flight Control: A Benchmark Challenge, Lecture Notes in Control and Information Sciences, Vol. 399, Springer-Verlag, Berlin/Heidelberg.

Edwards, C. and Spurgeon, S. (1998). Sliding Mode Control, Theory and Applications, Taylor and Francis, London.

Hamayun, M. (2013). Integral Sliding Mode Fault Tolerant Control Schemes with Control Allocation, Ph.D. thesis, University of Leicester, Leicester.

Hamayun, M., Edwards, C. and Alwi, H. (2012). Design and analysis of an integral sliding mode fault tolerant control scheme, IEEE Transactions on Automatic Control 57(7): 1783-1789.

Hamayun, M., Edwards, C. and Alwi, H. (2013). An output integral sliding mode FTC scheme using control allocation, Automatica 49(6): 1830-1837.

Harkegard, O. and Glad, S. (2005). Resolving actuator redundancy-optimal vs. control allocation, Automatica 41(1): 137-144.

Hess, R. and Wells., S. (2003). Sliding mode control applied to reconfigurable flight control design, Journal of Guidance, Control and Dynamics 26(3): 452-462.

Johansen, T. and Fossen, T.I. (2013). Control allocation-a survey, Automatica 49(5): 1087-1103.

Khalil, H. (1992). Nonlinear Systems, Prentice Hall, Englewood Cliffs, NJ.

Marcos, A. and Balas, G. (2003). A Boeing 747-100/200 aircraft fault tolerant and diagnostic benchmark, Technical report, Department of Aerospace and Engineering Mechanics, University of Minnesota, Minneapolis, MN.

Ogata, K. (2002). Modern Control Engineering, 4th Edn., Prentice Hall, London.

Shtessel, Y., Buffington, J. and Banda, S. (2002). Tailless aircraft flight control using multiple time scale re-configurable sliding modes, IEEE Transactions on Control Systems Technology 10(2): 288-296.

Utkin, V., Guldner, J. and Shi, J. (1999). Sliding Mode Control in Electromechanical Systems, Taylor and Francis, London.

Utkin, V. and Shi, J. (1996). Integral sliding mode in systems operating under uncertainty conditions, 35th IEEE Conference on Decision and Control, Kobe, Japan, pp. 4591-4596.

Verhaegen, M., Kanev, S., Hallouzi, R., Jones, C., Maciejowski, J. and Smail, H. (2010). Fault tolerant flight control-a survey, in C. Edwards, T. Lombaerts and H. Smaili (Eds.), Fault Tolerant Flight Control, Lecture Notes in Computer Science, Vol. 399, Springer, Berlin/Heidelberg, pp. 47-89.

Wells, S. and Hess, R. (2003). Multi-input/multi-output sliding mode control for a tailless fighter aircraft, Journal of Guidance, Control, and Dynamics 26(3): 463-473.

Wu, N., Zhang, Y. and Zhou, K. (2000). Detection, estimation, and accommodation of loss of control effectiveness, International Journal of Adaptive Control and Signal Processing 14: 775-795.
Zhang, Y. and Jiang, J. (2003). Fault tolerant control system design with explicit consideration of performance degradation, IEEE Transactions on Aerospace and Electronic Systems 39(3): 838-848.

Zhang, Y. and Jiang, J. (2008). Bibliographical review on reconfigurable fault-tolerant control systems, Annual Reviews in Control 32(2): 229-252.

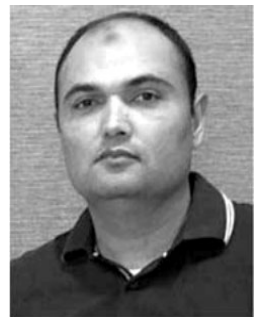

Mirza Tariq Hamayun was born in Pakistan $\mathrm{He}$ graduated from Eastern Mediterranean University, TRNC Turkey, with a B.Sc. and an M.Sc (Hons) in electrical engineering in 1999 and 2001, respectively, supported by EMU Turkey In 2001 he joined the Daimler Chrysler AG Research Centre, Frankfurt, Germany. He was awarded a Ph.D. from Leicester University in 2013, supported by a CIIT scholarship. He is a co-author of over 20 refereed papers. Currently he works as an assistant professor in the Department of Electrical Engineering, CIIT, Lahore. His research interests include sliding mode control and observation and their applications to fault tolerant control.

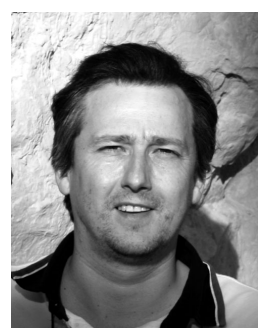

Christopher Edwards is a professor of control engineering in the College of Engineering, Mathematics and Physical Sciences at the University of Exeter, UK. His current research interests are in sliding mode control and observation, and their application to fault detection and fault tolerant control problems. He is the author of 300 refereed papers in these areas and three books: Sliding Mode Control: Theory and Applications (1998), Fault Detection and Fault Tolerant Control Using Sliding Modes (2011) and Sliding Mode Control and Observation (2013). In addition, he recently co-edited the monograph Fault Tolerant Flight Control: A Benchmark Challenge (2010).

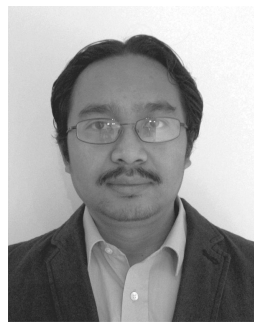

Halim Alwi was born in Malaysia. He studied at the University of Leicester and graduated in 2000 with a B.Eng. (Hons) in mechanical engineering. In 2000-2004 he was an engineer at The New Straits Times Press. In 2004 he moved back to the University of Leicester and was awarded a $\mathrm{Ph} . \mathrm{D}$. in 2008 in the area of fault tolerant control applied to aerospace systems. He is a co-author of over 60 refereed papers and a monograph on sliding mode control. He is currently a lecturer at the College of Engineering, Mathematics and Physical Sciences, University of Exeter.

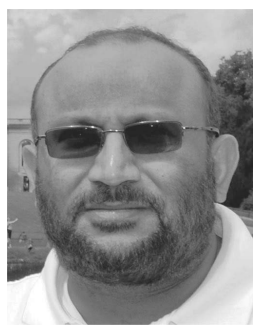

Abdulrahman Bajodah is a faculty member of the Aeronautical Engineering Department at King Abdulaziz University in Jeddah, Saudi Arabia. He received his B.Sc. degree in aeronautical engineering from King Abdulaziz University in 1992, and his Master's and Ph.D. degrees in aerospace engineering from the Georgia Institute of Technology in 1997 and 2003. His research interests include analytical mechanics, nonlinear and optimal control, and adaptive control systems, with applications to robotics, aircraft, and spacecraft control.

Received: 30 January 2014 Revised: 18 June 2014 